\title{
Cremona special sets of points in products of projective spaces
}

Igor V. Dolgachev

To the memory of V.I. Arnol'd

\begin{abstract}
A set of points in the projective plane is said to be Cremona special if its orbit with respect to the Cremona group of birational transformations consists of finitely many orbits of the projective group. This notion was extended by A. Coble to sets of points in higher-dimensional projective spaces and by S. Mukai to sets of points in the product of projective spaces. No classification of such sets is known in these cases. In the present article we survey Coble's examples of Cremona special points in projective spaces and initiate a search for new examples in the case of products of projective spaces. We also extend to the new setting the classical notion of associated points sets.
\end{abstract}

Keywords Coxeter group, Cremona transformations, associated sets of points. Mathematics Subject Classification (2010) Primary 14E07. Secondary 14N05, 11 H55.

\section{Introduction}

Let $W_{p, q, r}$ denote the Coxeter group defined by the Coxeter graph of type $T_{p, q, r}$.

We will be interested in the cases when the group $W_{p, q, r}$ is infinite. It follows from the classification of finite reflection groups that $W_{p, q, r}$ is infinite if and only if

\section{Igor V. Dolgachev}

Department of Mathematics, University of Michigan,

Ann Arbor, MI 48109, USA

e-mail: idolga@umich.edu 


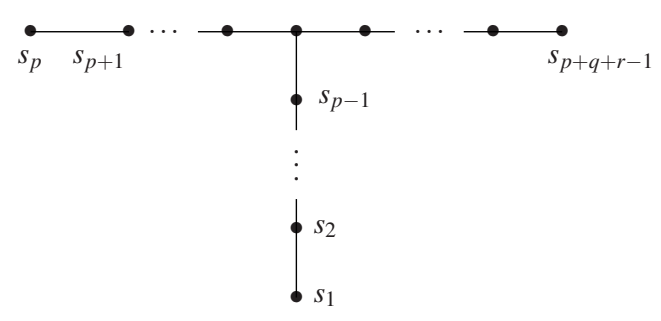

$\frac{1}{p}+\frac{1}{q}+\frac{1}{r} \leq 1$. Finite groups $W_{p, q, r}$ are known to be isomorphic to the Weyl groups of root systems of type $A_{2} \times A_{1}, A_{n}, D_{5}, E_{6}, E_{7}, E_{8}$.

It has been known since the fundamental work of A. Coble [2], [4] that the groups $W_{2, q, r}$ act birationally on the configuration spaces of ordered $q+r$ points in $\mathbb{P}^{q}$ modulo projective transformations. Roughly speaking, the subgroup generated by the generators $s_{p}, \ldots, s_{p+q+r-1}$ acts by permutations of points and the additional generator $s_{p-1}$ acts via the standard Cremona transformation of degree $q$ which inverts the coordinates of each point. The stabilizer subgroup of the orbit of a points set can be interpreted as a group of pseudo-automorphisms of the variety obtained by blowing-up this set. Here a pseudo-automorphism means a birational automorphism which is an isomorphism outside of a closed subset of codimension $\geq 2$. For example, if $q=2$, the group of automorphisms of the blow-up surface becomes isomorphic to a subgroup of the Coxeter group $W_{2,3, r}$. This result, sometimes attributed to M. Nagata [22], goes back to S. Kantor [18], A. Coble [2], and P. Du Val [12].

It is known that, for a general points set, the automorphism group of the blow-up surface is trivial [15], but in a special case it could be very large in the sense that it is realized as a subgroup of finite index in $W_{2,3, r}$. Coble was the first to initiate a search of points sets in projective spaces which are special in the sense that the pseudo-automorphism group of the blow-up variety is realized as a subgroup of finite index in $W_{2, q, r}$. For example, a set of points in the projective plane is special if its orbit with respect to the Cremona group contains only finitely many orbits with respect to the projective group. Coble gave several examples of special sets in the plane and in three-dimensional space. Among these examples is the set of base points of a pencil of plane cubic curves, or the set of nodes of a rational plane sextic, or the set of nodes of a Cayley symmetroid quartic surface. A modern treatment of Coble's theory can be found in [9].

A generalization of the Coble representation of $W_{2, q, r}$ in the group of birational automorphisms of $X_{2, q, r}$ to a representation of an arbitrary Coxeter group $W_{p, q, r}$ on the configuration spaces of ordered points sets in the product of projective spaces was recently given by S. Mukai [21]. He applied this to the construction of new 
counter-examples to Hilbert's 14th Problem about finite generation of rings of invariants. The purpose of the paper is to initiate a search of special sets of points in this new setting. We also extend the classical notion of association of points sets in projective space to points sets in the product of projective spaces.

\section{The Cremona action of $W_{p, q, r}$}

We define the configuration space of ordered sets of $q+r$ points in the product of projective spaces $\mathbb{P}_{p, q}:=\left(\mathbb{P}^{q-1}\right)^{q+r}$ to be the GIT-quotient

$$
X_{p, q, r}=\left(\mathbb{P}_{p, q}\right)^{q+r} / / \operatorname{SL}(q)^{p-1},
$$

where the group $\operatorname{SL}(q)^{p-1}$ acts naturally on the product $\mathbb{P}_{p, q}$ and diagonally on the product $\left(\mathbb{P}_{p, q}\right)^{q+r}$. We choose a democratic linearization on the product $\left(\mathbb{P}_{p, q}\right)^{q+r} \cong\left(\mathbb{P}^{q-1}\right)^{(p-1)(q+r)}$ defined by the invertible sheaf $\mathscr{O}(1)^{\bigotimes(p-1)(q+r)}$. To exclude the trivial cases, we assume that $p, q, r>1$. The variety $X_{p, q, r}$ is an irreducible rational variety of dimension $D=(p-1)(q-1)(r-1)$.

The group $\mathfrak{S}_{p-1} \times \mathfrak{S}_{q+r}$ acts naturally on $X_{p, q, r}$ by permuting the $p-1$ factors of $\mathbb{P}_{p, q}$ and $q+r$ factors of $\left(\mathbb{P}_{p, q}\right)^{q+r}$. It is realized as the subgroup of $W_{p, q, r}$ generated by the Coxeter generators $s_{1}, \ldots, s_{p-2}$ and $s_{p}, \ldots, s_{p+q+r-1}$. Following Coble, Mukai extends this action to a homomorphism

$$
\operatorname{cr}_{p, q, r}: W_{p, q, r} \rightarrow \operatorname{Bir}\left(X_{p, q, r}\right) \cong \operatorname{Aut}_{\mathbb{C}}\left(\mathbb{C}\left(z_{1}, \ldots, z_{D}\right)\right)
$$

by defining the action of the remaining generator $s_{p-1}$ and checking that the relations between the generators are preserved. Recall that the standard Cremona transformation $T$ in projective space $\mathbb{P}^{q-1}$ is a birational transformation given by the formula

$$
z=\left[z_{0}, \ldots, z_{q}\right] \mapsto\left[z_{0}^{-1}, \ldots, z_{q}^{-1}\right] .
$$

We extend $T$ to the product $\left(\mathbb{P}^{q-1}\right)^{p-1}$ by the formula

$$
\left(z^{(1)}, \ldots, z^{(p-1)}\right) \mapsto\left(\left[\frac{1}{z_{0}^{(1)}}, \ldots, \frac{1}{z_{q-1}^{(1)}}\right],\left[\frac{z_{0}^{(2)}}{z_{0}^{(1)}}, \ldots, \frac{z_{q-1}^{(2)}}{z_{q-1}^{(1)}}\right], \ldots,\left[\frac{z_{0}^{(p-1)}}{z_{0}^{(1)}}, \ldots, \frac{z_{q-1}^{(p-1)}}{z_{q-1}^{(1)}}\right]\right) .
$$

A general points set can be represented in $X_{p, q, r}$ by a unique ordered points set $\left(p_{1}, \ldots, p_{q+r}\right)$ with the first $q+1$ points equal to the reference points

$$
[1,0, \ldots, 0], \ldots,[0, \ldots, 0,1],[1, \ldots, 1] \text {. }
$$


We use projective transformations in each copy of $\mathbb{P}^{q-1}$ to assume that the projections of the first $q+1$ points $p_{1}, \ldots, p_{q+1}$ are the reference points in $\mathbb{P}^{q-1}$. Then we apply $T$ to the rest of the points. Note that $T$ is not defined at the intersections of the pull-backs of coordinate hyperplanes in the first factor $\mathbb{P}^{q-1}$. One checks that this action of generators preserves the defining relations of the Coxeter group and defines the homomorphism (1). We call this homomorphism the Cremona action of $W_{p, q, r}$.

Let $\mathscr{P}$ be an ordered set of $q+r$ distinct points in $\mathbb{P}_{p, q}$ and let

$$
\pi_{\mathscr{P}}: V_{\mathscr{P}} \rightarrow X^{q+r}
$$

be its blow-up. We consider $V_{\mathscr{P}}$ up to a birational isomorphism which is an isomorphism outside a closed subset of codimension $\geq 2$ (a pseudo-isomorphism). The action of such a birational isomorphism on the group $H^{2}\left(V_{\mathscr{P}}, \mathbb{Z}\right) \cong \operatorname{Pic}\left(V_{\mathscr{P}}\right)$ is welldefined. Denote by $E_{i} \cong \mathbb{P}^{p q-p-q}$ the exceptional divisor over the point $p_{i}$ and let $e_{i}=\left[E_{i}\right]$ be its class in $H^{2}\left(V_{\mathscr{P}}, \mathbb{Z}\right)$. Let $\mathrm{pr}_{i}: \mathbb{P}_{p, q} \rightarrow \mathbb{P}^{q-1}$ be the projection to the $i$-th factor and $h_{i}=\left[\pi^{*}\left(\operatorname{pr}_{i}^{*}(H)\right)\right]$, where $H$ is a hyperplane in $\mathbb{P}^{q-1}$. Then

$$
h_{1}, \ldots, h_{p-1}, e_{1}, \ldots, e_{q+r}
$$

form a basis of $H^{2}\left(V_{\mathscr{P}}, \mathbb{Z}\right)$. We call it a geometric basis. Let

$$
h^{1}, \ldots, h^{p-1}, e^{1}, \ldots, e^{q+r}
$$

be its dual basis in $H_{2}\left(V_{\mathscr{P}}, \mathbb{Z}\right)$. We can realize it by taking $-e^{i}$ to be the class of a line in $E_{i}$ and $h^{i}$ to be the homology class of a line in $\mathbb{P}^{q-1}$ embedded in $\mathbb{P}_{p, q}$ under the inclusion map in the $i$-th factor $l_{i}: \mathbb{P}^{q-1} \rightarrow\left(\mathbb{P}^{q-1}\right)^{p-1}$. Let

$$
\begin{aligned}
\alpha_{1} & =h_{p-2}-h_{p-1}, \ldots, \alpha_{p-2}=h_{1}-h_{2}, \\
\alpha_{p-1} & =h_{1}-e_{1}-\ldots-e_{q}, \\
\alpha_{p} & =e_{1}-e_{2}, \ldots, \alpha_{p+q+r-2}=e_{q+r-1}-e_{q+r}
\end{aligned}
$$

and

$$
\begin{aligned}
\alpha^{1} & =-h^{p-2}+h^{p-1}, \ldots, \alpha^{p-2}=-h^{1}+h^{2}, \\
\alpha^{p-1} & =(q-2) h^{1}+(q-1) h^{2}+\ldots+(q-1) h^{p-1}+e^{1}+\ldots+e^{q}, \\
\alpha^{p} & =e^{2}-e^{1}, \ldots, \alpha^{p+q+r-2}=-e_{q+r-1}+e_{q+r} .
\end{aligned}
$$

We immediately check that the matrix $\left(\alpha_{i}, \alpha^{j}\right)+2 I_{p+q+r-2}$ is the incidence matrix of the graph $T_{p, q, r}$. The two bases 


$$
\underline{\alpha}=\left(\alpha_{1}, \ldots, \alpha_{p+q+r-2}\right), \underline{\alpha}^{\vee}=\left(\alpha^{1}, \ldots, \alpha^{p+q+r-2}\right)
$$

form a root basis. The Weyl group of this root basis acts on $H^{2}\left(V_{\mathscr{P}}, \mathbb{Z}\right)$ (resp. on $\left.H_{2}\left(V_{\mathscr{P}}, \mathbb{Z}\right)\right)$ as the group generated by the simple reflections

$$
s_{i}: x \rightarrow x+\left(x, \alpha^{i}\right) \alpha_{i}\left(\text { resp. } s^{i}: y \rightarrow y+\left(y, \alpha_{i}\right) \alpha^{i}\right) .
$$

Let $e=e_{1}+\ldots+e_{q+r}$. It is immediately checked that

$$
\begin{aligned}
& 1 \leq i \leq p-2: s_{i}\left(h_{i}\right)=h_{i+1}, s\left(h_{j}\right)=h_{j}, j \neq i, i+1, \\
& s_{i}\left(e_{j}\right)=e_{j}, j=1, \ldots, q+r, \\
& i=p-1: s_{i}\left(h_{1}\right)=(q-1) h_{1}-(q-2) e, \\
& s_{i}\left(h_{j}\right)=h_{j}+(q-1)\left(h_{1}-e\right), j \neq 1, \\
& s_{i}\left(e_{j}\right)=h_{1}-e+e_{j}, 1 \leq j \leq q, s_{i}\left(e_{j}\right)=e_{j}, j>q . \\
& i>p: s_{i}\left(h_{j}\right)=h_{j}, j=1, \ldots, p-1, \\
& s_{i}\left(e_{p-1+i}\right)=e_{p+i}, s_{i}\left(e_{j}\right)=e_{j}, j \neq p-1+i, p+i .
\end{aligned}
$$

It follows that the Weyl group is isomorphic to the Coxeter group $W_{p, q, r}$ with Coxeter generators $s_{i}$.

Note that the following vectors are preserved under the action:

$$
\begin{aligned}
K_{V_{\mathscr{P}}} & =-q\left(h_{1}+\ldots+h_{p-1}\right)+(p q-p-q)\left(e_{1}+\ldots+e_{q+r}\right) \in H^{2}\left(V_{\mathscr{P}}, \mathbb{Z}\right), \\
k_{V_{\mathscr{P}}} & =-q\left(h^{1}+\ldots+h^{p-1}\right)+e^{1}+\ldots+e^{q+r} \in H_{2}\left(V_{\mathscr{P}}, \mathbb{Z}\right) .
\end{aligned}
$$

One recognizes in $K_{V_{\mathscr{P}}}$ the canonical class of $V_{\mathscr{P}}$. One can check that $\underline{\alpha}$ is a basis of the orthogonal complement of $k_{V_{\mathscr{P}}}$ in $H^{2}\left(V_{\mathscr{P}}, \mathbb{Z}\right)$ and $\underline{\alpha}^{\vee}$ is a basis of the orthogonal complement of $K_{V_{\mathscr{P}}}$ in $H_{2}\left(V_{\mathscr{P}}, \mathbb{Z}\right)$.

Another way to look at this is to define a linear map

$$
H^{2}\left(V_{\mathscr{P}}, \mathbb{Z}\right) \rightarrow H_{2}\left(V_{\mathscr{P}}, Z\right), \gamma \rightarrow \gamma^{\vee}
$$

on the basis $\left(h_{i}, e_{j}\right)$ by

$$
h_{i} \mapsto(q-1)\left(h^{1}+\ldots+h^{p-1}\right)-h^{i}, i=1, \ldots, p-1, \quad e_{i} \mapsto-e^{i}, i=1, \ldots, q+r .
$$

Then $x \cdot y:=\left(x, y^{\vee}\right)$ defines a structure of a quadratic lattice on $H^{2}\left(V_{\mathscr{P}}, \mathbb{Z}\right)$ with the Gram matrix in the basis $\left(h_{1}, \ldots, h_{p-1}, e_{1}, \ldots, e_{q+r}\right)$ given by block-sum of the square matrix 


$$
A_{p, q}:=\left(\begin{array}{ccccc}
q-2 & q-1 & q-1 & \ldots & q-1 \\
q-1 & q-2 & q-1 & \ldots & q-1 \\
\vdots & \vdots & \vdots & \vdots & \vdots \\
q-1 & q-1 & q-1 & \ldots & q-2
\end{array}\right)
$$

and the matrix $-I_{q+r}$. The signature of this lattice is equal to $(1, p+q+r-2)$ and the discriminant is equal to $(-1)^{q+r}(p q-p-q)$. We have

$$
K_{V_{\mathscr{P}}}^{\vee}=(p q-p-q) k_{V_{\mathscr{P}}}
$$

so $k_{V_{\mathscr{P}}}^{\perp}$ is mapped onto $K_{V_{\mathscr{P}}}^{\perp}$ and

$$
K_{V_{\mathscr{P}}}^{2}=(p q-p-q)(p q r-p q-p r-q r) .
$$

This implies that the sublattice $k_{V_{\mathscr{P}}}^{\perp}$ with a basis $\underline{\alpha}$ is an even lattice of signature $\left(t_{+}, t_{-}\right)\left(\right.$or $\left(t_{+}, t_{-}, t_{0}\right)$ if $\left.t_{0} \neq 0\right)$ :

$$
\operatorname{sign}\left(\mathbb{E}_{p, q, r}\right)= \begin{cases}(0, p+q+r-2) & \text { if } p q r-p q-p r-q r>0, \\ (0, p+q+r-3,1) & \text { if } p q r-p q-p r-q r=0, \\ (1, p+q+r-3) & \text { otherwise. }\end{cases}
$$

The group $W_{p, q, r}$ is finite if and only if $p q r-p q-p r-q r>0$ and it contains an abelian subgroup of finite index if and only if $p q r-p q-p r-q r=0$.

It is convenient to introduce an abstract quadratic lattice $\mathbf{I}_{p, q, r}$ defined in a basis $\left(\mathbf{h}_{1}, \ldots, \mathbf{h}_{p-1}, \mathbf{e}_{1}, \ldots, \mathbf{e}_{q+r}\right)$ by the matrix $A_{p, q} \oplus-I_{q+r}$, a vector

$$
\mathbf{K}_{p, q, r}=-q\left(\mathbf{h}_{1}+\ldots+\mathbf{h}_{p-1}\right)+(p q-p-q)\left(\mathbf{e}_{1}+\ldots+\mathbf{e}_{q+r}\right),
$$

and the sublattice

$$
\mathbb{E}_{p, q, r}:=\left(\mathbf{K}_{p, q, r}\right)^{\perp}
$$

with a canonical basis $\left(\alpha_{1}, \ldots, \alpha_{p+q+r-2}\right)$, where $\alpha_{i}$ are expressed in terms of $\left(\mathbf{h}_{1}, \ldots, \mathbf{h}_{p-1}, \mathbf{e}_{1}, \ldots, \mathbf{e}_{q+r}\right)$ by formulas (4). We also define the Weyl group $W\left(\mathbb{E}_{p, q, r}\right)$ as the group of orthogonal transformations of the lattice $\mathbb{E}_{p, q, r}$ generated by reflections with respect to the vectors $\alpha_{i}$ :

$$
s_{i}: x \mapsto x+\left(x \cdot \alpha_{i}\right) \alpha_{i}
$$

This is the Coxeter group corresponding to the $T_{p, q, r}$-graph. For simplicity of notation, we set $W\left(\mathbb{E}_{p, q, r}\right)=W_{p, q, r}$. 
A choice of a geometric basis in $H^{2}\left(V_{\mathscr{P}}, \mathbb{Z}\right)$ defines a geometric marking of $V_{\mathscr{P}}$, i.e. an isometry of lattices $\varphi: \mathbf{I}_{p, q, r} \rightarrow H^{2}\left(V_{\mathscr{P}}, \mathbb{Z}\right)$ such that $\varphi\left(\mathbf{K}_{p, q, r}\right)=K_{V_{\mathscr{P}}}$. Under this isometry the lattice $\mathbb{E}_{p, q, r}$ is mapped onto $K_{V_{\mathscr{P}}}^{\perp}$.

It is easy to see that $W_{p, q, r}$ acts transitively on the root basis $\underline{\alpha}$ of $\mathbb{E}_{p, q, r}$. An element of the $W_{p, q, r}$-orbit of any element from the canonical basis is called a root. A root is called positive if it can be written as a linear combination of the canonical basis with non-negative coefficients. One can show that any root $\alpha$ is either positive or $-\alpha$ is positive.

Lemma 2.1 Let $\quad \alpha=\sum_{i=1}^{p-1} d_{i} \mathbf{h}_{i}-\sum_{j=1}^{q+r} m_{i} \mathbf{e}_{j}$ be a positive root. Let $d=d_{1}+\ldots+d_{p-1}$. Suppose that one of the numbers $d_{i}$ is positive. Then

(i) $q d=\sum_{j=1}^{q+r} m_{j}$;

(ii) $(q-1) d^{2}-\sum_{i=1}^{p-1} d_{i}^{2}-\sum_{k=1}^{q+r} m_{k}^{2}=-2$;

(iii) $(q-1) d-d_{1}<m_{1}+\ldots+m_{q}$, if $m_{1} \geq m_{2} \geq \ldots \geq m_{q+r}$ and $d_{1} \leq \ldots \leq d_{p-1}$;

(iv) assume $d>0$, then $d_{i} \geq 0, i=1, \ldots, p-1$, and $m_{j} \geq 0, j=1, \ldots, q+r$.

Proof The first equality follows from the condition that $\alpha \cdot \mathbf{K}_{p, q, r}=0$. The second one follows from the condition that $\alpha^{2}=-2$.

(iii) The ordering of the coefficients implies that $\alpha \circ \alpha_{i} \geq 0$ for $i \neq p-1$. One checks that

$$
\alpha \cdot \alpha_{p-1}=(q-1) d-d_{1}-\sum_{j=1}^{q} m_{j} .
$$

Assume the inequality does not hold. Then $\alpha \cdot \alpha_{i} \geq 0$ for all $\alpha_{i}$. This means that $\alpha$ belongs to the fundamental chamber of the root system. The proof that it is impossible is the same as in the proof of the corresponding statement for the case $p=2$ in [9], p. 75.

(iv) It is checked immediately that $d \geq 0$ for a positive root. Let us use induction on $d$. Assume $d=1$. Then (i) and (ii) give

$$
1-\sum_{j=1}^{p-1} d_{i}^{2}=\sum_{j=1}^{q} m_{j}\left(m_{i}-1\right) .
$$

The left-hand side is non-positive, the right-hand-side is non-negative. This gives that one of the $d_{i}$ is equal to 1 , all others are zeros. Also, $m_{j}=1$ or 0 . This checks the assertion. 
It is known that $s_{i}$ transforms the set of positive roots with $\alpha_{i}$ deleted to a subset of positive roots (see [17], Lemma 1.3). Applying $s_{i}, i \neq p-1$, we may assume that that $d_{i}$ 's and $m_{j}^{\prime}$ are ordered as in (iii). Using (5), we obtain

$$
\alpha^{\prime}=s_{p-1}(\alpha)=\sum_{i=1}^{p-1} d_{i}^{\prime} \mathbf{h}_{i}-\sum_{j=1}^{q+r} m_{i}^{\prime} \mathbf{e}_{j}
$$

where

$$
\begin{aligned}
& d_{1}^{\prime}=(q-1) d-\sum_{j=1}^{q} m_{j}, \\
& m_{j}^{\prime}=(q-1) d-d_{1}-\left(m_{1}+\ldots+m_{q}-m_{j}\right), j=1, \ldots, q,
\end{aligned}
$$

and all other $d_{i}$ 's and $m_{j}$ 's are unchanged. By (iii), we obtain that $d_{1}^{\prime}<d_{1}$, and hence $d^{\prime}=\sum d_{i}^{\prime}<d$. If $\sum d_{i}^{\prime}>0$, we are done by induction. Assume that $d^{\prime}=0$. Then (i) and (ii) give $2-\sum d_{i}^{2}=\sum m_{j}^{2}$. This gives the following possibilities $\alpha^{\prime}=h_{i}-h_{j}, e_{i}-e_{j}, i<j$. But then $\alpha=s_{p-1}\left(\alpha^{\prime}\right)$ satisfies (iv) and (v).

Note that a vector in $\mathbf{I}_{p, q, r}$ satisfying (i)-(iv) from the lemma is not necessarily a root. An example in the case $p=2, q=3$ is given in [9].

Lemma 2.2 Let $\alpha$ be a root in $\mathbb{E}_{p, q, r}$ and $\Delta(\alpha)$ be the subset of $\mathbb{P}_{p, q}$ that consists of points sets such that $\varphi_{\mathscr{P}}(\alpha)$ is effective. Then $\Delta(\alpha)$ is a closed proper subset of $\mathbb{P}_{p, q}$.

Proof If $\alpha=\alpha_{i}$ or its transform under $s_{i}, i \neq p-1$, the assertion is obvious. The root $\alpha_{i}, i<p-1$, is never effective, the roots $\alpha_{i}, i>p-1$, are effective only if some points coincide, and $\alpha_{p-1}$ is effective only if the first $q+1$ points are in a hyperplane. A points set for which all such roots are not effective will be called a regular point set. It follows from Lemma 2.1 that, for roots $\alpha$ with $d=\sum d_{i}>0$, the condition that its image is effective reads as the condition of the existence of a hypersurface of multi-degree $\left(d_{1}, \ldots, d_{p-1}\right)$ passing through the points $p_{i} \in \mathscr{P}$ with multiplicity $\geq m_{i}$. Obviously it is a closed condition. Assume $\Delta(\alpha)=\mathbb{P}_{p, q}$ for some $\alpha$ with $\sum d_{i}>0$. Without loss of generality, we may assume that $m_{1} \geq \ldots \geq m_{q+r}$ and $d_{1} \leq \ldots \leq d_{p-1}$. Also we may assume that $\mathscr{P}$ is a regular set. Now we take $\mathscr{Q}$ from the projective equivalence class of $\operatorname{cr}_{p, q, r}\left(s_{p-1}\right)([\mathscr{P}])$. Then $\varphi_{\mathscr{Q}}\left(s_{p-1}(\alpha)\right)$ is an effective root (the transform under $T$ of the hypersurface defining $\varphi_{\mathscr{P}}(\alpha)$ ). Property (iii) of a positive root together with (6) implies that the hypersurface defined by the root $\varphi_{\mathscr{Q}}\left(s_{p-1}(\alpha)\right)$ has multi-degree $\left(d_{1}^{\prime}, \ldots, d_{p-1}^{\prime}\right)$ with $d^{\prime}=\sum d_{i}^{\prime}<d$. Now we can use induction on $d$. We know that $\Delta\left(s_{p-1}(\alpha)\right)$ is a proper subset, hence we find a regular $\mathscr{Q}$ such that the root $s_{p-1}(\alpha)$ is not effective. 
Definition $1 \mathrm{~A}$ points set $\mathscr{P}$ is called unnodal if under the geometric marking $\varphi_{\mathscr{P}}: \mathbf{I}_{p, q, r} \rightarrow \operatorname{Pic}\left(V_{\mathscr{Q}}\right)$ defined by $\mathscr{P}$, no root is mapped to an effective divisor class.

It follows from Lemma 2.2 that the set of unnodal points sets is the complement of the union of proper closed subsets $\Delta(\alpha)$, where $\alpha$ is a positive root in $\mathbb{E}_{p, q, r}$. This set is infinitely countable if the lattice $\mathbb{E}_{p, q, r}$ is not negatively definite.

Note that we do not know whether the closed subsets $\Delta(\alpha)$ are hypersurfaces in $\mathbb{P}_{p, q}$ when $\alpha$ is a root different from $\alpha_{i}, i \neq p-1$. It is true in the cases when $\mathbb{P}_{p, q}$ is a surface.

Proposition 1 Let $\mathscr{P}$ be an unnodal point set. Then, for any $w \in W_{p, q, r}$, the marking ${ }^{w} \varphi:=\varphi \circ w^{-1}$ is a geometric marking on $V_{\mathscr{P}}$ defined by the points set $\mathscr{Q}$ such that $\operatorname{cr}_{p, q, r}([\mathscr{P}])=[\mathscr{Q}]$.

Proof Let $\mathscr{P}$ be a regular set of points. We use that, for Coxeter generator $s_{i} \in W_{p, q, r}, \operatorname{cr}_{p, q, r}\left(s_{i}\right)$ is defined at $[\mathscr{P}]$. If $s_{i} \neq s_{p-1}$, then ${ }^{s_{i}} \varphi_{\mathscr{P}}=\varphi_{\mathscr{Q}}$, where $\mathscr{Q}$ is obtained from $\mathscr{P}$ by either permuting the points, or as the image of an automorphism of $\mathbb{P}_{p, q}$ permuting the $(p-1)$ factors. If $i=p-1$, then [Q2] is defined by the points set (3), where $T$ is the standard Cremona transformation of $\mathbb{P}_{p, q}$. Note that the divisor classes $E_{i}^{\prime}$ representing $s_{p-1}\left(e_{i}\right), i=1, \ldots, q$, are not contractible on $V_{\mathscr{P}}$ if $\operatorname{dim} \mathbb{P}_{p, q}>2$. However, they become contractible when we apply to $V_{\mathscr{P}}$ a pseudoisomorphism. The geometric marking corresponding to a points set $\mathscr{Q}$ representing $\operatorname{cr}_{p, q, r}\left(s_{i}\right)([\mathscr{P}])$ is equal to ${ }^{s_{i}} \varphi$. So, if we show that $\mathscr{Q}$ is again a regular set, we are done.

Let $\mathfrak{l}(w)$ be the minimal length of $w$ as the product of Coxeter generators. This is well-defined in any Coxeter group. Let us prove by induction on $\mathfrak{l}(w)$ that the image of a geometric basis defined by $\mathscr{P}$ under $w$ is a geometric basis. Write $w$ as the product of generators $s_{i_{k}} \cdots s_{i_{1}}$, where $k=\mathfrak{l}(w)$. Assume $i_{1} \neq p-1$. Then, as we have already observed, $s_{i_{1}}$ transforms a geometric basis to a geometric basis. It is defined by a regular points set $\mathscr{Q}$ such that $\operatorname{cr}_{p, q, r}\left(s_{i_{1}}\right)([\mathscr{P}])=[\mathscr{Q}]$.

Assume $i_{1}=p-1$. Consider the birational transformation $\operatorname{cr}_{p, q, r}\left(s_{p-1}\right)$ of $X_{p, q, r}$. It transforms the point $[\mathscr{P}]$ corresponding to a normalized points set $\mathscr{P}=\left(p_{1}, \ldots, p_{q+1}, \ldots, p_{q+r}\right)$ to the point $[\mathscr{Q}]$, where

$$
\mathscr{Q}=\left(p_{1}, \ldots, p_{q+1}, T\left(p_{q+2}\right), \ldots, T\left(p_{q+r}\right)\right)=\left(p_{1}^{\prime}, \ldots, p_{q+r}^{\prime}\right) .
$$

Assume that $\mathscr{Q}$ is not regular. If it does not satisfy the first condition of regularity, then some points in this set coincide. But this could happen only if one of the points $p_{i}, i>q+1$, in $\mathscr{P}$ lies in an exceptional divisor of $T$ (i.e. the closure of 
points which are mapped to the set of indeterminacy of $T^{-1}=T$ ). It is easy to see that this set consists of the union of the pre-images of hyperplanes in the first copy of $\mathbb{P}^{q-1}$ which are spanned by all points $\operatorname{pr}_{1}\left(p_{1}\right), \ldots, \operatorname{pr}_{1}\left(p_{q+1}\right)$ except one, say $p_{j}$. Since $h_{1}-e_{1}-\ldots-e_{q}+e_{j}-e_{i}$ is not an effective divisor, the images of the points $\left\{p_{1}, \ldots, p_{q}, p_{i}\right\} \backslash\left\{p_{j}\right\}$ under the first projection are not contained in a hyperplane. So the new points set $\mathscr{Q}$ consists of distinct points. Next assume that the second condition of regularity is not satisfied. This means that the projections of some points $p_{j_{1}}^{\prime}, \ldots, p_{j_{q+1}}^{\prime}$ to some $t$-factor lie in a hyperplane. Applying $\mathrm{cr}_{p, q, r}\left(s_{i}\right), 1 \leq i \leq p-2$, we may assume that $t=1$. It follows from the definition of the transformation $T$ that $T^{*}\left(h_{1}\right)=2 h_{1}-e_{1}-\ldots-e_{q}$. It agrees with the action of $s_{p-1}$ on the geometric basis. If the points $p_{j_{1}}^{\prime}, \ldots, p_{j_{q+1}}^{\prime}$ are projected to a hyperplane in the first factor, then the pre-image of this hyperplane under $T$ is an effective divisor in the class

$$
2 h_{1}-e_{1}-\ldots-e_{q}-e_{j_{1}}-\ldots-e_{j_{q+1}}=s_{p-1}\left(h_{1}-e_{j_{1}}-\ldots-e_{j_{q+1}}\right) \text {, }
$$

where we replace $e_{j_{k}}$ with $e_{i}$ if $j_{k}=i$ for some $i \leq q$. Since $h_{1}-e_{j_{1}}-\ldots-e_{j_{q+1}}$ is a root, and $s_{p-1}\left(h_{1}-e_{j_{1}}-\ldots-e_{j_{q+1}}\right)$ is again a root, it cannot be effective. This proves that $s_{i_{1}}$ is well defined on $[\mathscr{P}]$ and transforms it to [Q⿻], where $\mathscr{Q}$ is a regular point set. It remains to apply induction on $\mathfrak{l}(w)$.

Proposition 2 Let $\mathscr{P}$ be an unnodal points set and $w \in W_{p, q, r}$ be such that $\operatorname{cr}_{p, q, r}(w)([\mathscr{P}])=[\mathscr{P}]$. Then there exists a pseudo-automorphism $\tau: V_{\mathscr{P}} \rightarrow V_{\mathscr{P}}$ such that $w=\varphi_{\mathscr{P}}^{-1} \circ \tau^{*} \circ \varphi_{\mathscr{P}}$.

Proof Let $\sigma: V_{\mathscr{P}} \rightarrow \mathbb{P}_{p, q}$ be a birational morphism contracting the divisors $E_{1}, \ldots, E_{q+r}$ to points $p_{1}, \ldots, p_{q+r} \in \mathscr{P}$. Then there exists a pseudo-automorphism $\Phi: V_{\mathscr{P}}-\rightarrow V_{\mathscr{P}}^{\prime}$ and a contraction $\sigma^{\prime}: V_{\mathscr{P}}^{\prime} \rightarrow \mathbb{P}_{p, q}$ of divisors $E_{i}^{\prime}$ to the same set of points $\mathscr{P}$ such that $\Phi^{*}\left(E_{i}^{\prime}\right)=\varphi_{\mathscr{P}} \circ w \circ \varphi_{\mathscr{P}}^{-1}$. Since two blow-ups of the same closed subscheme are isomorphic, there exists an isomorphism $\Psi: V_{\mathscr{P}} \rightarrow V_{\mathscr{P}}^{\prime}$ which sends $E_{i}$ to $E_{i}^{\prime}$. The composition $\tau=\Phi \circ \Psi^{-1}: V_{\mathscr{P}} \rightarrow V_{\mathscr{P}}$ is a pseudoautomorphism of $V_{\mathscr{P}}$ whose existence is asserted in the Proposition.

Corollary 1 Let $\mathscr{P}$ be an unnodal set in $\mathbb{P}_{p, q}$. Then the stabilizer subgroup

$$
\left(W_{p, q, r}\right)_{\mathscr{P}}:=\left\{w \in W_{p, q, r}: \operatorname{cr}_{p, q, r}(w)([\mathscr{P}])=[\mathscr{P}]\right\}
$$

is isomorphic to a subgroup of the group $\mathrm{Aut}_{\mathrm{ps}}\left(V_{\mathscr{P}}\right)$ of the group of pseudoautomorphisms of $V_{\mathscr{P}}$.

Let $\Phi \in \operatorname{Aut}_{\mathrm{ps}}\left(V_{\mathscr{P}}\right)$ and let $\Phi^{*}$ be its action on $H^{2}\left(V_{\mathscr{P}}, \mathbb{Z}\right)$. We say that $\Phi$ is Cremona-like if there exists $w \in W_{p, q, r}$ such that 


$$
w=\varphi_{\mathscr{P}}^{-1} \circ \Phi^{*} \circ \varphi_{\mathscr{P}} .
$$

Let $\operatorname{Aut}_{\mathrm{cr}}\left(V_{\mathscr{P}}\right)$ be the subgroup of $\operatorname{Aut}_{\mathrm{ps}}\left(V_{\mathscr{P}}\right)$ of Cremona-like transformations. We have a natural homomorphism

$$
\operatorname{Aut}_{\mathrm{cr}}\left(V_{\mathscr{P}}\right) \rightarrow W_{p, q, r}, \Phi \mapsto \varphi_{\mathscr{P}}^{-1} \circ \Phi^{*} \circ \varphi_{\mathscr{P}}
$$

It is clear that its kernel is isomorphic to the group of automorphisms of $V_{\mathscr{P}}$ lifted from automorphisms of $\mathbb{P}_{p, q}$.

Remark 1 In the case $p=2, q=3$, one can prove, using Noether's Theorem on generators of the planar Cremona group, that $\operatorname{Aut}_{\mathrm{cr}}\left(V_{\mathscr{P}}\right)=\operatorname{Aut}\left(V_{\mathscr{P}}\right)$.

It seems that A. Coble [2] and S. Kantor [19] claimed that $\operatorname{Aut}_{\mathrm{cr}}\left(V_{\mathscr{P}}\right)=\operatorname{Aut}_{\mathrm{ps}}\left(V_{\mathscr{P}}\right)$ in the cases $p=2$ and $q$ is arbitrary. Their claim was based on their theory of punctual or regular Cremona transformation of $\mathbb{P}^{q-1}$. A punctual Cremona transformation is a product of projective transformations and the standard Cremona transformations. By Noether's Theorem, any planar Cremona transformation is punctual. Kantor says that a Cremona transformation $\Phi$ of $\mathbb{P}^{3}$ has no fundamental curves of the 1 st kind if the graph of $\Phi$ transforms any fundamental curve to a fundamental curve of the inverse transformation. He claimed that any such transformation is punctual (citing from [16], p. 318: "the so-called proof is admittedly "gewagt", and merits a stronger adjective"). Another "equivalent definition" ([13], p. 192) of a punctual transformation requires that all base conditions follow from conditions at points. No rigorous proof of equivalence of these definitions is available. What we need is to prove (or disprove) the following assertion:

Let $\sigma_{i}: V_{\mathscr{P}_{i}}-\rightarrow \mathbb{P}_{p, q}$ be two blow-up varieties and $\Phi: V_{\mathscr{P}_{1}}-\rightarrow V_{\mathscr{P}_{2}}$ be a pseudo-isomorphism. Then the Cremona transformation $\sigma_{2} \circ \Phi \circ \sigma_{1}^{-1}: \mathbb{P}_{p, q}-\rightarrow \mathbb{P}_{p, q}$ is a composition of the standard transformation (3) and automorphisms of $\mathbb{P}_{p, q}$.

\section{Examples of Cremona special sets}

Definition 2 A points set $\mathscr{P}$ is called Cremona special if the image $\operatorname{Aut}_{\mathrm{cr}}\left(V_{\mathscr{P}}\right)^{*}$ of $\operatorname{Aut}_{\mathrm{cr}}\left(V_{\mathscr{P}}\right)$ in $W_{p, q, r}$ is a subgroup of finite index. 
Since the condition is vacuous when $W_{p, q, r}$ is a finite group, we will additionally assume that $W_{p, q, r}$ is an infinite group.

All examples of Cremona special sets that I know use the theory of abelian fibrations that I remind below.

Let $Y$ be a smooth projective variety over a field $L$. There exists an abelian variety $\operatorname{Alb}^{0}(Y)$ and a torsor $\operatorname{Alb}^{1}(Y)$ over $\operatorname{Alb}^{0}(Y)$ satisfying the universal property for morphisms of $Y$ to torsors over an abelian variety [23], [25]. If $Y(K) \neq \emptyset$, then $\operatorname{Alb}^{0}(Y)=\operatorname{Alb}^{1}(Y)$ is the Albanese variety $\operatorname{Alb}(Y)$ of $Y$. The dual abelian variety of $\operatorname{Alb}^{0}(Y)$ is isomorphic to the (reduced) Picard variety $\mathbf{P i c}_{Y / K}^{0}$. We say that $Y$ is an abelian torsor if the canonical map $Y \rightarrow \operatorname{Alb}^{1}(Y)$ is an isomorphism. In the simplest case when $\operatorname{dim} Y=1$, this means that $Y$ is a smooth curve of genus 1 , and $\operatorname{Alb}^{0}(Y)=\mathbf{P i c}_{Y / K}^{0}$ is its Jacobian variety.

Let $f: X \rightarrow S$ be a projective morphism of irreducible varieties. We assume that $S$ is smooth and $X$ is $\mathbb{Q}$-factorial with terminal singularities. Assume that a generic fibre $X_{\eta}$ is an abelian torsor over the field of rational functions $K=\kappa(\eta)$ of $S$. Moreover, assume that $f$ is relatively minimal in the sense that $K_{X} \cdot C \geq 0$ for any curve $C$ contained in a fibre. Let $\mathbf{A}=\operatorname{Alb}^{1}\left(X_{\eta}\right)$, the group $\mathbf{A}(K)$ acts biregularly on $X_{\eta}$ via translations

$$
t_{a}: x \mapsto x+a, \quad x \in X_{\eta}(\bar{K}), a \in \mathbf{A}(K)
$$

This action defines a birational action on $X$, and, the condition of minimality implies that the action embeds $\mathbf{A}(K)$ into the group $\operatorname{Aut}_{\mathrm{ps}}(X)$ of pseudo-automorphisms of $X$ [20], [24], Lemma 6.2.

Assume that the class group $\mathrm{Cl}(X)$ of Weyl divisors is finitely generated of rank $\rho$ (e.g. $X$ is a unirational variety as it will be in all applications). Let

$$
r_{\eta}: \mathrm{Cl}(X) \rightarrow \mathrm{Cl}\left(X_{\eta}\right) \cong \operatorname{Pic}\left(X_{\eta}\right)
$$

be the restriction homomorphism. By taking the closure of any point of codimension 1 in $X_{\eta}$, we see that this homomorphism is surjective. Let $\operatorname{Pic}^{0}\left(X_{\eta}\right)=\operatorname{Pic}_{X_{\eta} / K}^{0}(K)$ be the subgroup of Cartier divisor classes of algebraically equivalent to zero. Then we have an exact sequence of finitely generated abelian groups

$$
0 \rightarrow \mathrm{Cl}_{\mathrm{fib}}(X) \rightarrow \mathrm{Cl}(X)^{0} \rightarrow \operatorname{Pic}\left(X_{\eta}\right)^{0} \rightarrow 0,
$$

where $\mathrm{Cl}_{\mathrm{fib}}(X)$ is the subgroup of $\mathrm{Cl}(X)$ generated by the classes of Weil divisors $\sum n_{i} D_{i}$ such that $\operatorname{codim} f\left(D_{i}\right) \geq 1$. Since the dual abelian varieties are isogenous, we obtain that the group $\mathbf{A}(K)$ is finite generated. In the case when $X_{\eta}(K) \neq \emptyset$, 
hence $X_{\eta} \cong \mathbf{A}$, the group $\mathbf{A}(K)$ is called the Mordell-Weil group of the fibration. We keep this terminology in the general case and denote the group $\mathbf{A}(K)$ by $\operatorname{MW}(f)$. Counting the ranks, we obtain the Shioda-Tate formula

$$
\operatorname{rank} \mathrm{MW}(f)=\operatorname{rank} \mathrm{Cl}(X)-1-\operatorname{rank} \mathrm{Cl}(S)-\sum_{s \in S^{(1)}}\left(\# \operatorname{Irr}\left(X_{S}\right)-1\right),
$$

where $S^{(1)}$ is the set of points of codimension 1 in $S$ and $\operatorname{Irr}\left(X_{S}\right)$ is the set of irreducible components of the fibre $X_{S}$.

Example 1 Assume $p=2, q=3$, hence $r \geq 6$. The known examples in these cases are due to A. Coble [1], [4]. The first example is an Halphen set of index $m$, the set of 9 base points of an irreducible pencil of curves of degree $3 m$ with nine $m$-multiple base points (an Halphen pencil of index $m$ ). The proper transform of the pencil in $V_{\mathscr{P}}$ is the linear system $\left|-m K_{V_{\mathscr{P}}}\right|$. We assume that all fibres are irreducible which is equivalent to the condition that the set $\mathscr{P}$ is unnodal. When $m=1$ this can be achieved by assuming that no three points are collinear ([24], Lemma 3.1). Let $C$ be the unique cubic curve passing through the base points $p_{1}, \ldots, p_{9}$. We assume that $C$ is nonsingular. Obviously, this is an open condition on $\mathscr{P}$. If we fix a group law on $C$ with an inflection point as the zero, then the condition that $\mathscr{P}$ is an Halphen set of index $m$ is that the points $p_{i}$ add up in the group law to a point of order $m$. Let

$$
a: \mathbb{Z}^{9} \rightarrow C, \quad\left(m_{1}, \ldots, m_{9}\right) \mapsto m_{1} p_{1}+\ldots+m_{9} p_{9},
$$

where the sum is taken in the group law on $C$. Assume that $a(\mathbf{m}) \neq 0$ for any vector $\mathbf{m}=\left(m_{1}, \ldots, m_{9}\right)$ with $\left(m-m_{1}, \ldots, m-m_{9}\right) \in \mathbb{Z}_{>0}^{9}$ and $m_{1}+\ldots+m_{9}<2 m$. This is an open condition on $\mathscr{P}$ that guarantees that there are no curves of degree $d<2 m$ with singular points at the point $p_{i}$ of multiplicity $m_{i}$. Now, if $D$ is a reducible member of the pencil, one of its parts has degree $<2 m$ and passes through the $p_{i}$ with some multiplicity $m_{i}$ as above. By assumption, this is impossible, so all members of the pencil are irreducible, and stay the same when we blow-up the nine points. Since all $(-2)$-curves on $V_{\mathscr{P}}$ are contained in fibres, we obtain that $\mathscr{P}$ is unnodal. Let $f: V_{\mathscr{P}} \rightarrow \mathbb{P}^{1}$ be the elliptic fibration defined by the Halphen pencil. Applying the Shioda-Tate formula, we find that the rank of the Mordell-Weil group is equal to 8 . Thus $\mathbb{Z}^{8}$ embeds into $\operatorname{Aut}_{\mathrm{ps}}\left(V_{\mathscr{P}}\right)=\operatorname{Aut}\left(V_{\mathscr{P}}\right)$. The known structure of $W_{2,3,6}$ shows that the image is a normal subgroup of finite index. More detailed analysis of the action gives that the quotient group is isomorphic to $(\mathbb{Z} / 8 \mathbb{Z})^{8} \rtimes W_{2,3,5}$ (see [15]).

In the second example, $\mathscr{P}$ is a Coble set, the ten nodes $p_{1}, \ldots, p_{10}$ of an irreducible rational plane curve of degree 6 . The proper inverse transform $C$ of the 
sextic is equal to the anti-bicanonical linear system $\left|-2 K_{V_{\mathscr{P}}}\right|$. The double cover of $V_{\mathscr{P}}$ branched along $C$ is a $\mathrm{K} 3$-surface which can be considered as a degeneration of the étale double cover of an Enriques surface. One uses the period theory of Enriques surfaces to show that the locus of Coble sets is irreducible. Also, using some lattice-theoretical methods from [8], one can show that there are only finitely many closed conditions on $\mathscr{P}$ (in fact, exactly 496 conditions) that guarantee that $\mathscr{P}$ is an unnodal set. Let $\mathscr{C} o b \subset P_{2}^{10}$ be the subset of projective equivalence classes of unnodal Coble sets. This is a locally closed subset invariant with respect to the Cremona action. Let

$$
\mathrm{cr}_{2,3,7}: W_{2,3,7} \rightarrow \operatorname{Aut}(\mathscr{C} o b)
$$

be the action homomorphism. Its kernel is a normal subgroup of $W_{2,3,7}$. Observe that any subset of 9 points in $\mathscr{P}$ is an Halphen set of index 2, The Halphen pencil is generated by the sextic curve and the unique cubic curve through the 9 points taken with multiplicity 2. Conversely, a Coble set is obtained from an Halphen set of index 2 by choosing a singular member of the pencil, its singular point is the tenth point of the set. Fix two points, say $p_{1}$ and $p_{2}$ and let $F_{1}$ and $F_{2}$ be the proper transforms in $V_{\mathscr{P}}$ of the cubic curves through $p_{3}, \ldots, p_{10}$ and $p_{i}, i=1,2$. The linear system $\left|2 F_{i}\right|$ is a pencil, the proper transform of the corresponding Halphen pencil. We have $F_{i} \cdot F_{2}=1$ so that $\left(2 F_{1}+2 F_{2}\right)^{2}=8$. One can show that the linear system $\left|2 F_{1}+2 F_{2}\right|$ defines a degree two map from $V_{\mathscr{P}}$ onto a quartic Del Pezzo surface in $\mathbb{P}^{4}$ with 4 ordinary double points. The image of the curve $C$ is one of these nodes. The deck transformation of the cover defines an isometry of the lattice $K_{V_{\mathscr{P}}}^{\perp} \cong \mathbb{E}_{2,3,7}$ which is conjugate to the isometry $-\mathrm{id}_{\mathbb{E}_{2,3,5}} \oplus \mathrm{id}_{U}$, where $\mathbb{E}_{2,3,7} \cong U \oplus \mathbb{E}_{2,3,5}$ is an orthogonal decomposition into the sum of two unimodular sublattices. It is known that the normal subgroup of $W_{2,3,7}$ containing this isometry has finite quotient group isomorphic to $\mathrm{O}\left(10, \mathbb{F}_{2}\right)^{+}$[7], Theorem 2.10.1.

Recently, in a joint work with S. Cantat, we were able to prove the following.

Theorem 1 Let $\mathscr{P}$ be a Cremona special set in the plane. Then it is either an Halphen set, or a Coble set, or a set of $\geq 10$ points on an irreducible cuspidal curve in characteristic $p>0$.

Coble also constructed examples of Cremona special sets in $\mathbb{P}^{3}$. 
Example 2 The first series of examples are analogs of Halphen sets. One considers an elliptic normal curve $C$ of degree 4 in $\mathbb{P}^{3}$ equipped with the group law defined by a choice of an osculating point. Choose 8 points $\mathscr{P}=\left\{p_{1}, \ldots, p_{8}\right\}$ on $C$ in general position which add up to a point of order $m$ in the group law. Then one shows that there exists a surface $F_{2 m}$ of degree $2 m$ with $m$-multiple points at $p_{1}, \ldots, p_{m}$ which does not belong to the family of surfaces of the form $G_{m}\left(q_{1}, q_{2}\right)=0$, where $G_{m}$ is a homogeneous polynomial of degree $m$ and $q_{1}$ and $q_{2}$ are quadratic forms in 3 homogeneous coordinates in $\mathbb{P}^{3}$ such that $C=V\left(q_{1}\right) \cap V\left(q_{2}\right)$. The linear system $\left|\mathscr{O}_{\mathbb{P}^{3}}(2 m)-m\left(p_{1}+\ldots+p_{m}\right)\right|$ defines a regular map

$$
f: V_{\mathscr{P}} \rightarrow \mathbb{P}^{m+1}
$$

Its image is the projective cone over a Veronese curve of degree $m$ in $\mathbb{P}^{m}$. The image of $C$ is the vertex of the cone. If we fix a nonsingular quadric $Q$ in the pencil of quadrics $V\left(\lambda q_{1}+\mu q_{2}\right)$, then the restriction of the linear system to $Q$ is a pencil of elliptic curves of degree $4 m$ with eight $m$-multiple points. Thus a general fibre of $f$ is an elliptic curve. If we blow-up the proper transform of $C$ in $V_{\mathscr{P}}$, we obtain an elliptic fibration

$$
f^{\prime}: V_{\mathscr{P}}^{\prime} \rightarrow \mathbf{F}_{m}
$$

the fibres over the points on the exceptional section of $\mathbf{F}_{m}$ are $m$-multiple elliptic curves. The case $m=1$ corresponds to a well-known set of Cayley octads, the complete intersection of three quadrics. It is discussed in detail in [9]. By choosing the set $\mathscr{P}$ general enough, as in the example of Halphen sets, we find that all fibres over points of codimension 1 are irreducible. Since rank $\operatorname{Pic}\left(V_{\mathscr{P}}^{\prime}=10, \operatorname{rank} \operatorname{Pic}\left(\mathbf{F}_{m}\right)=2\right.$, applying the Shioda-Tate formula (2), we obtain that rank $\operatorname{MW}\left(f^{\prime}\right)=7$. Thus the kernel of $\operatorname{cr}_{2,4,4}$ contains a normal subgroup isomorphic to $\mathbb{Z}^{7}$. It is known to be a normal subgroup of $W_{2,4,4}$. If $m=1$, the quotient group is isomorphic to $W_{2,3,4}$. If $m>1$ it must be isomorphic to $(\mathbb{Z} / m \mathbb{Z})^{7} \rtimes W_{2,3,4}$; however, I confess that I have not checked this.

The second series of examples generalizes a Coble set. It is the set of 10 nodes of a symmetric determinantal quartic surface (Cayley symmetroid). This example has been worked out in detail in an unpublished manuscript [7] (see also [8]). The group of pseudo-automorphisms of $V_{\mathscr{P}}$ contains a normal subgroup of $W_{2,4,6}$ with quotient isomorphic to $\operatorname{Sp}\left(8, \mathbb{F}_{2}\right)$.

Here are some new examples in the cases $p>2$.

Example 3 Take $p=q=r=3$, so that $W_{3,3,3}$ is the affine Weyl group of type $E_{6}$. We are dealing with the set of 6 points in $\mathbb{P}^{2} \times \mathbb{P}^{2}$ modulo $\mathrm{PGL}_{3} \times \mathrm{PGL}_{3}$. Consider the 
subsets $\mathscr{P}=\left(x_{1}, \ldots, x_{6}\right)$ such that $\operatorname{dim}\left|h_{1}+h_{2}-x_{1}-\ldots-x_{6}\right|=3$ (one more than expected). It is known that this is equivalent to that the two projections of $\mathscr{P}$ to $\mathbb{P}^{2}$ are associated sets of 6 points in the plane [10]. It is known that the variety of such pairs modulo projective equivalence is isomorphic to an open subset of $\mathbb{P}^{4}$ [9]. Since $\left(h_{1}+h_{2}\right)^{4}=6$, for a general set $\mathscr{P}$, the linear system $L=\left|h_{1}+h_{2}-x_{1}-\ldots-x_{6}\right|$ has $\mathscr{P}$ as its set of base points, hence defines a morphism

$$
f: V_{\mathscr{P}} \rightarrow \mathbb{P}^{3}
$$

whose general fibre is the intersection of three divisors of type $(1,1)$, hence is an elliptic curve. The exceptional divisors $E_{1}, \ldots, E_{6}$ are disjoint sections of this fibration.

The restriction of $f$ over a general line $\ell$ in $\mathbb{P}^{3}$ defines an elliptic fibration $f_{\ell}: V_{\ell} \rightarrow \mathbb{P}^{1}$, where $V_{\ell}$ is the blow-up of 6 points in a complete intersection of two divisors in $\left|h_{1}+h_{2}\right|$ in $\mathbb{P}^{2} \times \mathbb{P}^{2}$. The latter is known to be isomorphic to a Del Pezzo surface of degree 6, the blow-up of three non-collinear points in the plane embedded into $\mathbb{P}^{8}$ by the linear system of plane cubics through the three points. Thus $f_{\ell}$ is an elliptic fibration obtained from an Halphen pencil of index 1.

Let $\Delta \subset \mathbb{P}^{3}$ be the locus of points $x \in \mathbb{P}^{3}$ such that $f^{-1}(x)$ is a singular fibre. Assume that $\mathscr{P}$ is general enough so that $\Delta$ is reduced. It follows from the theory of elliptic fibrations that reducible fibres $f^{-1}(x)$ over points of codimension 1 lie over singular points of $\Delta$ of codimension 1 . Thus, under our assumption all such fibres are irreducible. We may apply the Shioda-Tate formula to obtain that rank $\mathrm{MW}(f)=6$. Thus the action of the Mordell-Weil group defines a subgroup in the kernel of $\mathrm{cr}_{3,3,3}$ isomorphic to $\mathbb{Z}^{6}$. This is known to be a normal subgroup of finite index with quotient isomorphic to $W_{2,3,3}$. A subgroup of finite index has only finitely many orbits in the set of roots of $\mathbb{E}_{3,3,3}$. This shows that the infinitely many discriminant subvarieties of $X_{3,3,3}$ restrict to finite many subvarieties on the open set of points $[\mathscr{P}]$ such that the elliptic fibration (8) has reduced discriminant surface $\Delta$. It remains to check that the set of such points is open in the set of points [ $\mathscr{P}$ ] with $\operatorname{dim}\left|h_{1}+h_{2}-x_{1}-\ldots-x_{6}\right|=3$. For this it is enough to show that this set is non-empty. This can be done by explicit computation by taking a sufficiently general pair of associated sets of 6 points in the plane (see more about the association in the next section).

Example 4 Take $p=q=3, r=4$. We are dealing with the set of 7 points in $\mathbb{P}^{2} \times \mathbb{P}^{2}$ modulo $\mathrm{PGL}_{3} \times \mathrm{PGL}_{3}$. The linear system of hypersurfaces of bi-degree $(2,2)$ is of dimension 35. If we take 7 general points $p_{1}, \ldots, p_{7}$, then we expect a unique hypersurface with double points at the $p_{i}$ 's. However, there is a pencil $\mathscr{L}$ of hyper- 
surfaces of bi-degree $(1,1)$ through these points, so there will be a 2-dimensional linear system $\mathscr{N}$ of hypersurfaces of bi-degree $(2,2)$ with double points at points at $\mathscr{L}$. Let us consider a set $\mathscr{P}$ of 7 points such that there exists a hypersurface $Z$ of bi-degree $(2,2)$ with double points at $p_{1}, \ldots, p_{7}$ which does not belong to $\mathscr{N}$. Let $C$ be the intersection of $Z$ with the base surface of the pencil $\mathscr{L}$. It is a curve of arithmetic genus 7 with 7 double points, hence it is a rational curve. We have $-K_{V_{\mathscr{P}}}=3\left(h_{1}+h_{2}-e_{1}-\ldots-e_{6}\right)$, hence $[C]=\left(-\frac{2}{3} K_{V_{\mathscr{P}}}\right)^{3}$, so the locus of points $[\mathscr{P}]$ in $X_{3,3,4}$ with the above property is invariant with respect to the Cremona action.

Let $\mathscr{P}_{i}=\mathscr{P} \backslash\left\{p_{i}\right\}$. The linear system of hypersurfaces of bi-degree $(1,1)$ through $\mathscr{P}_{i}$ is of dimension 2 and the linear system of hypersurfaces of bi-degree $(2,2)$ is of dimension 6 and contains $Z$. Then we get a rational map

$$
f_{i}: V_{\mathscr{P}_{i}} \rightarrow \mathbb{P}^{6}
$$

with the image equal to the cone $S$ over the Veronese surface. Its fibres are elliptic curves, the proper transforms of curves of arithmetic genus 7 with 6 double points. The exceptional divisors $E_{i}$ are 2-sections of the elliptic fibration $f_{i}$. This is all similar to the example of a Coble set so that $f_{i}$ is an analog of the Halphen pencil of index 2 through 9 double points out of ten nodes of the Coble sextic. An elliptic curve $F$ over a field $K$ and a point $x_{0} \in F$ of degree 2 defines a degree 2 map $F \rightarrow \mathbb{P}^{1}$ over $K$. It is given by the linear series $\left|x_{0}\right|$. We apply this to our situation to obtain a birational involution $\sigma_{i}$ of $V_{\mathscr{P}_{i}}$ over $S$. This involution extends to the localization of $f_{i}$ over any point $s \in S^{(1)}$. This implies that $\sigma_{i}$ is a pseudo-automorphism of order 2 . This involution is defined at the point $p_{i}$ and extends to a pseudo-automorphism $\tilde{\sigma}_{i}$ of $V_{\mathscr{P}}$. Following the analogy with Coble sets, I speculate that any two involutions $\tilde{\sigma}_{i}, \tilde{\sigma}_{j}$ commute and the product $\sigma=\tilde{\sigma}_{i} \circ \tilde{\sigma}_{j}$ defines an involution of $K_{V_{\mathscr{P}}}^{\perp} \cong \mathbb{E}_{3,3,4}$ conjugate to the involution $-\mathrm{id}_{\mathbb{E}_{2,3,3}} \oplus \mathrm{id} U$, where $\mathbb{E}_{2,3,3}$ and $U$ are orthogonal summands of $\mathbb{E}_{3,3,4}$. Also I speculate that the normal subgroup of $W_{3,3,4}$ generated by this involution is of finite index in $W_{3,3,4}$ and generates the 2-level congruence subgroup $W_{3,3,4}(2)$. It will follow then that the quotient group is isomorphic to $\mathrm{O}\left(6, \mathbb{F}_{2}\right)^{-}$(see [7], Theorem 2.9.1).

\section{Association}

Examples of Cremona special sets of points in higher-dimensional spaces $\mathbb{P}^{n}$ can be obtained by the classical construction called the association (in modern time known as the Gale transform). It is discussed in detail in [9] or [14]. To give an idea, one 
considers a linear map $\mathbb{C}^{q+r+1} \rightarrow \mathbb{C}^{q+1}$ defined by the matrix with columns equal to projective coordinates of the points. The kernel of this linear map is isomorphic to $\mathbb{C}^{r+1}$, and the transpose map defines a map $\mathbb{C}^{q+r} \rightarrow \mathbb{C}^{r+1}$ representing $q+r$ points in $\mathbb{P}^{r}$. This is well-defined on the projective equivalence classes of ordered points sets. The association defines an isomorphism of GIT-quotients

$$
\text { as : } X_{2, q, r} \rightarrow X_{2, r, q} \text {. }
$$

The Coble action of $W_{2, p, q} \cong W_{2, q, r}$ commutes with the association so that the image of a Cremona special set in $\mathbb{P}^{q}$ is a Cremona special set in $\mathbb{P}^{r}$. However, to see explicitly the action is rather non-trivial geometric problem.

The following nice example is due to B. Totaro [24].

Example 5 Let $p_{1}, \ldots, p_{9}$ be the image of an Halphen set of index 1 under a Veronese map $\mathbb{P}^{2} \rightarrow \mathbb{P}^{5}$. This set is associated to the set $\mathscr{P}$ (see [3], Thm. 20, [11], Proposition 5.4). Consider the linear system of cubic hypersurfaces with double points at $p_{1}, \ldots, p_{9}$. Its dimension is equal to 3 and its base locus consists of 45 curves:36 lines through $p_{i}, p_{j}$, and 9 rational normal curves through all points except one. Let $V_{\mathscr{P}}-\rightarrow \mathbb{P}^{3}$ be the corresponding rational map. Its base locus consists of 45 disjoint $\mathbb{P}^{1}$ 's with normal bundle $\mathscr{O}_{\mathbb{P}^{1}}(-1)^{4}$. One can perform a flip on these curves giving another smooth 5 -fold $W$ in which the 45 curves are replaced with 45 smooth threedimensional subvarieties $S_{i}$ isomorphic to $\mathbb{P}^{3}$ with normal bundle $\mathscr{O}_{\mathbb{P}}(-1)^{2}$. Now we have a morphism $\Phi: W \rightarrow \mathbb{P}^{3}$ with general fibre isomorphic to an abelian surface. The subvarieties $S_{i}$ are sections and generate the Mordell-Weil group of rank 8. The exceptional divisors $E_{i}$ cut out on the fibres twice a principal polarization. The abelian fibration $\Phi$ is relatively minimal in the sense that $K_{W} \cdot C=0$ for any curve contained in a fibre. This implies that the translations by sections act on $W$ by pseudo-automorphism [20]. Thus $\operatorname{Aut}_{\mathrm{ps}}\left(V_{\mathscr{P}}\right) \cong \operatorname{Aut}_{\mathrm{ps}}(W)$ contains a subgroup isomorphic to $\mathbb{Z}^{8}$. It is known that the such a group must be of finite index in $W_{2,3,6}$.

The associated set of the set $\mathscr{P}=\left\{p_{1}, \ldots, p_{10}\right\}$ of 10 nodes of a quartic symmetroid surface is a set of 10 points in $\mathbb{P}^{5}$ which is equal to the intersection of two Veronese surfaces [3], Theorem 26. The secant variety of a Veronese surface is a cubic hypersurface singular along the surface. This shows that the associated set $\mathscr{Q}=\left\{q_{1}, \ldots, q_{10}\right\}$ is contained in the base locus of a pencil of cubic hypersurfaces with double points at the $q_{i}$ 's. The set of 9 points $\mathscr{Q}_{i}=\mathscr{Q} \backslash\left\{q_{i}\right\}$ is associated to the set $\Sigma_{i}$ of 9 points in the plane equal to the projection of the set $\mathscr{P}_{i} \backslash\left\{p_{i}\right\}$ from $p_{i}$ [9], Chapter 3, Prop. 4. It is known that the set $\Sigma_{i}$ is equal to the set of base points of a pencil of cubics. In fact, this property distinguishes Cayley symmetroids from other 10-nodal quartic surfaces [6]. Thus any subset of 9 points $\mathscr{Q}_{i}$ in $\mathscr{Q}$ is a set 
from Totaro's example. Let $G_{i} \cong \mathbb{Z}^{8}$ be the group of pseudo-automorphisms of $V_{\mathscr{Q}_{i}}$ defined by the Mordell-Weil of the corresponding abelian fibration. The point $p_{i}$ is a singular point of one of its fibres and is a fixed point of $G_{i}$. Thus $G_{i}$ extends to a group of pseudo-automorphisms of $V_{\mathscr{P}}$. One may ask whether the subgroups $G_{i}$ generate a subgroup of finite index in $W_{2,4,6}$. I do not know the answer.

Note that the permutation group $\mathfrak{S}_{3}$ acts on the Weyl group $W_{p, q, r}$ via permuting $(p, q, r)$. The relation between $X_{p, q, r}$ and $X_{p, r, q}$ is the product of $p-1$ copies of the association map. This defines an isomorphism as $23: X_{p, q, r} \rightarrow X_{p, r, q}$. The varieties $X_{q, p, r}$ and $X_{p, q, r}$ are not isomorphic but there exists a natural birational isomorphism. In fact, let us consider $X_{p, q, r}$ as the GIT-quotient of the product of $p-1$ copies of $\left(\mathbb{P}^{q-1}\right)^{q+r}$ by $\operatorname{PGL}(q)^{p-1}$. Using PGL $(q)$ in each copy we can fix the first $q+1$ points among $q+r$-points. The quotient becomes birationally isomorphic to $\left(\left(\mathbb{P}^{q-1}\right)^{r-1}\right)^{p-1}$, which is birationally isomorphic to $\mathbb{C}^{(p-1)(q-1)(r-1)}$. Now if we do the same with $X_{q, p, r}$ we obtain a birational model isomorphic to $\left(\left(\mathbb{P}^{p-1}\right)^{r-1}\right)^{q-1}$. which is birationally isomorphic to the same space $\mathbb{C}^{(p-1)(q-1)(r-1)}$.

Example 6 Consider the set of Cayley octads in $\mathbb{P}^{3}$ as a generalized Halphen set of index 1 . This is a Cremona special set for $W_{2,4,4}$. It is self-associated, with respect to the symmetry of the Dynkin diagram. Now consider the set of 6 points $q_{1}, \ldots, q_{6}$ in $\left(\mathbb{P}^{1}\right)^{3}$. It corresponds to $(p, q, r)=(4,2,4)$. Make the six points Cremona special by requiring that the linear system of divisors of type $(1,1,1)$ containing $\mathscr{P}$ is of dimension one larger than expected. The rational map given by this linear system defines an elliptic fibration $f: V_{\mathscr{P}} \rightarrow \mathbb{P}^{2}$. It has 6 disjoint sections defined by the exceptional divisors $E_{1}, \ldots, E_{6}$. Applying the Shioda-Tate formula, we obtain that the rank of the Mordell-Weil group is equal to 7. This is a subgroup of finite index in $W_{2,4,4}$.

Conjecture 1 Let $\sigma \in \mathfrak{S}_{3}$ and $\sigma: X_{p, q, r} \rightarrow X_{\sigma(p), \sigma(q), \sigma(r)}$ be the birational map described above. Then

$$
\operatorname{cr}_{\sigma(p), \sigma(q), \sigma(r)}=\sigma \circ \mathrm{cr}_{p, q, r} \circ \sigma^{-1} .
$$

It follows from this conjecture that the image of a Cremona special set of points under the $\sigma$-association is a Cremona special set of points. 


\section{References}

1. A. Coble, The ten nodes of the rational sextic and of the Cayley symmetroid, Amer. J. Math. 41 (1919), 243-265.

2. A. Coble, Point sets and allied Cremona groups. II. Trans. Amer. Math. Soc. 17 (1916), $345-$ 385 .

3. A. Coble, Associated sets of points. Trans. Amer. Math. Soc. 24 (1922), 1-20.

4. A. Coble, Algebraic geometry and theta functions (reprint of the 1929 edition), A. M. S. Coll. Publ., v. 10. A. M. S., Providence, R.I., 1982.

5. A. Coble, A Generalization of the Weddle Surface, of Its Cremona Group, and of Its Parametric Expression in Terms of Hyperelliptic Theta Functions. Amer. J. Math. 52 (1930), 439-500.

6. F. Cossec, Reye congruences. Trans. Amer. Math. Soc. 280 (1983), 737-751.

7. F. Cossec, I. Dolgachev, On automorphisms of nodal Enriques surfaces. Bull. Amer. Math. Soc. (N.S.) 12 (1985), 247-249.

8. F. Cossec, I. Dolgachev, On automorphisms of nodal Enriques surfaces, unpublished manuscript.

9. I. Dolgachev, D. Ortland, Point sets in projective spaces and theta functions. Astérisque, $\mathbf{1 6 5}$ (1988).

10. I. Dolgachev, M. Kapranov, Arrangements of hyperplanes and vector bundles on $\mathbf{P}^{n}$. Duke Math. J. , 71 (1993), 633-664.

11. I. Dolgachev, On certain families of elliptic curves in projective space. Ann. Mat. Pura Appl. (4) $\mathbf{1 8 3}$ (2004), no. 3, 317-331.

12. P. Du Val, On the Kantor group of a set of points in a plane, Proc. London Math. Soc. 42 (1936), 18-51.

13. P. Du Val, Crystallography and Cremona transformations. "The Geometric Vein", pp. 191201, Springer, New York-Berlin, 1981.

14. D. Eisenbud, S. Popescu, The projective geometry of the Gale transform. J. Algebra 230 (2000), no. 1, 127-173.

15. M. Gizatullin, Rational G-surfaces. Izv. Akad. Nauk SSSR Ser. Mat. 44 (1980), 110-144.

16. H. Hudson, Cremona transformations in plane and space, Cambridge Univ. Press. 1927.

17. V. Kac, Infinite-dimensional Lie algebras. Third edition. Cambridge University Press, Cambridge, 1990.

18. S. Kantor, Theorie der endlichen Gruppen von eindeutigen Transformationen in der Ebene, Berlin. Mayer \& Müller. 1895.

19. S. Kantor, Theorie der Transformationen im $R_{3}$ welche keine fundamental Curven 1. Art besitzen, Acta Math. 21 (1897), 1-78.

20. Y.Kawamata, Flops connect minimal models, Publ. RIMS Kyoto Univ., 44 (2008), 419-423.

21. S. Mukai, Geometric realization of T-shaped root systems and counterexamples to Hilbert's fourteenth problem, in "Algebraic transformation groups and algebraic varieties", 123-129, Encyclopaedia Math. Sci., 132, Springer, Berlin, 2004.

22. M. Nagata, On rational surfaces. II. Mem. Coll. Sci. Univ. Kyoto Ser. A Math. 33 (1960/1961), 271-293.

23. J.-P. Serre, Morphismes universels et variétés d'Albanese, Exposés de séminaires, 1950-1989, Soc. Math. France, 2001, 141-160.

24. B. Totaro, Hilbert's 14th problem over finite fields and a conjecture on the cone of curves. Compos. Math. 144 (2008), 1176-1198.

25. O. Wittenberg, On Albanese torsors and the elementary obstruction. Math. Ann. 340 (2008), $805-838$. 